\title{
The Accurate Measurement of a Micromechanical Force Using Force-Sensitive Capacitances
}

\author{
M. R. Wolffenbuttel and P. P. L. Regtien
}

\begin{abstract}
A sensor for the precise and accurate measurement of micromechanical forces is presented. The sensor is based on the capacitive detection of force-induced deflection of a microstructure and integrated charge readout. The mechanical performance of the sensor is evaluated and verified with experiments. The structure has been designed to enable the separation of the forceto-deflection and deflection measurements in order to result in a sensor structure in which the response is linear with force and little affected by fringe fields. The sensor is $0.25 \mathrm{~mm}^{2}$ in size and has a force range of $0.2 \mathrm{mN}$, a zero-force capacitance of $0.5 \mathrm{pF}$, a sensitivity of $1-5 \mathrm{fF} / \mu \mathrm{N}$, and a resolution that corresponds to a capacitance variation of $2 \mathrm{fF}$.
\end{abstract}

\section{INTRODUCTION}

A TACTILE image sensor is used for the acquisition of information about shapes of an object by touching it. The sensor has a pressure-sensitive surface to measure the distribution of small contact forces, and tactile information is obtained with a matrix of force-sensitive cells, which are arranged in rows and columns. The elements are the capacitors, which are formed at each row-column intersection so that touch can be detected with on-chip charge amplification. The tactile data can, for example, be used to monitor the position of an object during the act of touch, and additional pattern recognition algorithms can be employed to extract details of particular objects.

This paper describes the basic sensor structure that is suitable to detect a small mechanical force. The sensor has been realized in a surface micromachining process and involves the deposition and the etching of silicon nitride and polysilicon structural layers on a silicon wafer [1]. The process combines sensor fabrication and IC technology and leads to sensors with on-chip electronic circuits. Small sensor elements with a high sensitivity can be fabricated. Measurement results on practical devices are presented, and the performance of the sensor is shown to be in agreement with theory.

\section{SENSOR OPERATION}

The layout of a force-sensing element presented here is based on a design that is optimized with respect to mapping of force range on the available dynamic range of the sensor

Manuscript received July I, 1994; revised October 15, 1994. This work was supported by the Dutch Foundation for Fundamental Research on Matter (FOM) and the Netherlands Technology Foundation (STW)

M. R. Wolffenbuttel is with the Department of Electrical Engineering, Delft University of Technology, 2628 CD Delft, The Netherlands.

P. P. L. Regtien is with the Department of Electrical Engineering, University Twente, 7500 AE Enschede. The Netherlands.

IEEE Log Number 9408833.
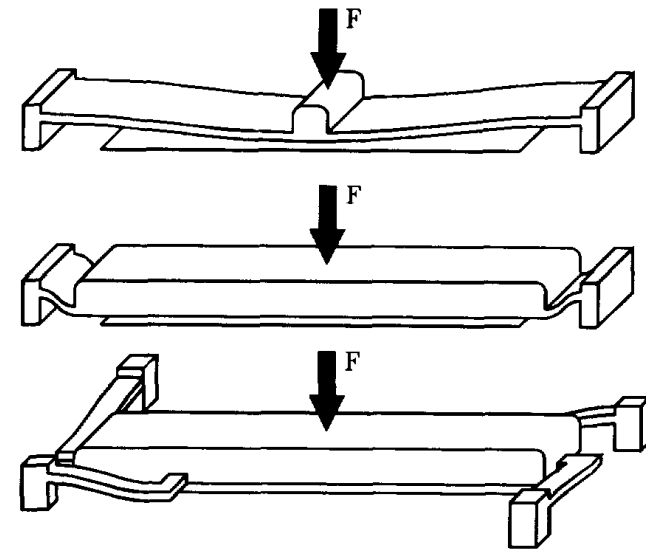

Fig. 1. Typical layouts of a capacitive force sensor.

capacitance at a specified maximum nonlinearity. In a typical mechanical sensor, shown in Fig. 1(a), a micromachined beam performs two functions. First, the beam displaces under an applied load and functions as a mechanical spring. Second, the beam is an electrode and forms a capacitance with the substrate, which changes in value. The main disadvantage of this configuration is that the elastic bending curve of the electrode load is not uniform. Displacements in the different beam sections are not according to simple analytic expressions and complex numerical calculations are therefore required to evaluate the applied microforce from the measured capacitance.

This limitation can generally be circumvented using an advanced bossed structure [2] that overlays the entire capacitive sensor electrode, shown in Fig. 1(b). The thicker central part of a beam ensures a uniform narrowing of the capacitive gap with applied load, and the determination of this force can be accurately calculated from a simple plane parallelplate capacitance approximation. A sensor can be further improved by designing a structure that gives an independent setting of the sensor capacitance, e.g., the electrode area and membrane-to-substrate spacing, and the force range, e.g., the geometric dimensions and strength against buckling of the spring elements. This setup is shown in Fig. 1(c) and features a sensor that has a force range and a sensitivity that can accurately and independently be adapted for different ranges without the need for a critical rescaling of the sensor. The design of the sensor in this project is based on this approach. Fig. 2 shows a representation of the sensor under a load 


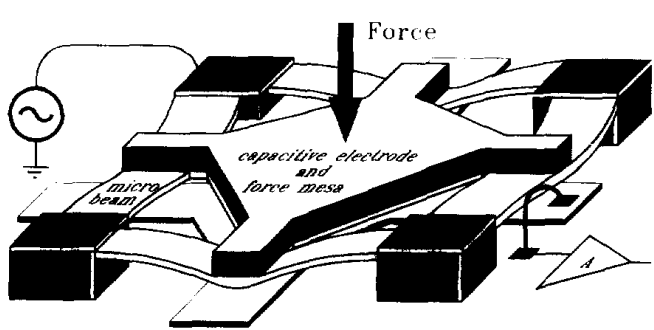

Fig. 2. The force sensor.

and is basically a tandem transducer of a mechanical force into an electrical capacitance via an intermediate mechanical displacement.

The sensor capacitance at zero applied force, which is generally referred to as a nominal sensor capacitance $C_{o}$, is determinated by the capacitance area $A$ and membrane-tosubstrate spacing $d_{o}$. Micromechanical fabrication processes yield very small membranes, but capacitances that are realized can be considerable when the gap is very narrow. Typically, the membrane area is $150 \times 150 \mu \mathrm{m}^{2}$ and the capacitive gap in the submicron range, resulting in a capacitance in the $0.1-2 \mathrm{pF}$ region [3]. A micromechanical force decreases the capacitive gap between the membrane and the substrate and increases the sensor capacitance. Changes in the sensor capacitance due to an applied force can thus be simply detected.

\section{THEORY}

The sensor performance has been theoretically analyzed, and mechanical parameters such as force range, sensitivity, and resolution are evaluated. A practical range for the sensor can be defined as the force that reduces the gap separation between the membrane and the substrate by a factor of two, and by definition, the cell capacitance doubles from $C_{o}$ to $2 C_{0}$. From the theory of mechanics, the differential equation of a deflecting beam oriented along the $x$-axis is given by $E I \cdot d^{4} y / d x^{4}+T \cdot d^{2} y / d x^{2}=F(x)$, with $F(x)$ the force distribution over the beam, $I$ the moment of inertia of a beam section, $E$ the modulus of elasticity of the beam material, $T$ the axial load, and $y$ the direction of deflection [4]. The force range and sensitivity of the sensor, which is shown in Fig. 2, are given by

$$
\begin{gathered}
F_{\max } \approx \frac{192\left(\left.4 E I\right|_{b}\right) d_{o} \alpha}{2 L^{3}} \\
S_{\text {cap }}=\frac{\partial C}{C_{o} \partial F} \approx \frac{d_{o} L^{3}}{\left(d_{o}-y\right)^{2} 192\left(\left.4 E I\right|_{b}\right) \alpha}
\end{gathered}
$$

with

$E=$ modulus of elasticity of the beam.

$I=$ moment of inertia of a beam section.

$\left.E I\right|_{b}=$ stiffness of beam: $\left.E I\right|_{b}=2 \times 10^{-13} \mathrm{Nm}^{2}$.

$d_{o}=$ initial capacitive gap spacing: $d_{0}=0.5 \mu \mathrm{m}$.

$\alpha=$ correction for internal stress: $\alpha=1020$.

$L=$ beam length: $L=100-150 \mu \mathrm{m}$.
Typically, $F_{\max }=0.1-0.5 \mathrm{mN}$ and $\partial C / \partial F=1-5 \mathrm{fF} / \mu \mathrm{N}$. Equation (2) shows that the sensor response has a nonlinear characteristic with applied force. The maximum sensitivity at $d=d_{o} / 2$ is four times the force sensitivity at zero force. The maximum operating force and the sensitivity can be scaled within a range of magnitudes by changing sensor dimensions like beam width, beam length, and gap spacing between the membrane and the substrate.

The force resolution is the smallest force that can be resolved. Noise, offset, and drift are some typical phenomena that determine this limit. Electrical noise from a readout circuit has been demonstrated to be the detection limit [5]. The charge amplifier resolves a minimum capacitance change of $\Delta C_{\mathrm{min}}$ and the force resolution $R_{F}$ is

$$
R_{F}=\frac{\Delta C_{\mathrm{min}}}{C_{o} \cdot S_{\text {cap }}}=\frac{192\left(\left.4 E I\right|_{b}\right) \alpha \Delta C_{\mathrm{min}}}{\varepsilon_{o} A} \frac{d_{o}^{2}}{L^{3}} .
$$

Typically, $\Delta C_{\min }=2 \mathrm{fF}, C_{\circ}=0.5 \mathrm{pF}$, and $\left.R_{F}\right|_{F}=0=1$ $-5 \mu \mathrm{N}$. This value can be compared with the maximum force of $0.1-0.5 \mathrm{mN}$ and enables the division of a force range in 128 reproducible levels.

\section{Results}

Surface micromachined force-sensitive structures were realized. The sensor has a typical dimension of $0.5 \mu \mathrm{m}$ thick and $150 \mu \mathrm{m}$ wide. Fig. 3 shows a SEM detailed view of this structure, which has a calculated force range $F_{\max }=0.28$ $\mathrm{mN}$, a sensor capacitance of $0.6 \mathrm{pF}$, and an area of $0.5 \mathrm{~mm}^{2}$. To examine the agreement of the theory with experiments, an electrostatic voltage was applied across the gap between a free-standing sensor membrane and the substrate. Electrostatic normal forces cause the structure to deflect [6]. If the force exceeds the total normal component of the stress induced in the beams, the structure pulls in and collapses. The mechanical contact forces are basically replaced by another, noncontact force in this technique, which simplifies the mechanical testing.

Fig. 4 shows a schematic overview of the different capacitances involved in the experiment. The sensor capacitance $C_{s}$ is connected in series with a dielectric capacitor $C_{d}$, which is formed by the substrate oxide/nitride insulating layers. Another important capacitance is junction capacitance $C_{j}$, which is parallel to $C_{s}$ and formed between the lower capacitance plate and the silicon substrate. Fig. 5 shows the measured capacitance as a function of applied voltage. $C_{j}$ decreases with increasing voltage up to about $15 \mathrm{~V}$, and the sensor capacitance remains essentially unchanged. The measured capacitance is approximately equal to the junction capacitance, and the figure shows a drop in this curve due to the extending depletion layer. The sensor capacitance changes abruptly when the bridge collapses and a corresponding pull-in voltage, $V_{\mathrm{pi}}=23 \mathrm{~V}$, can be accurately determined. With decreasing voltage, a pullout voltage, $V_{\text {po }}=18 \mathrm{~V}$, can be detected, which indicates that the membrane releases from the substrate [6].

A measurement of $V_{\mathrm{pi}}$ and $V_{\mathrm{po}}$ can be employed to determine $F_{\max }$ and $C_{d}$. Fig. 6 shows the forces that act on the membrane as a function of applied voltage over the sensor 


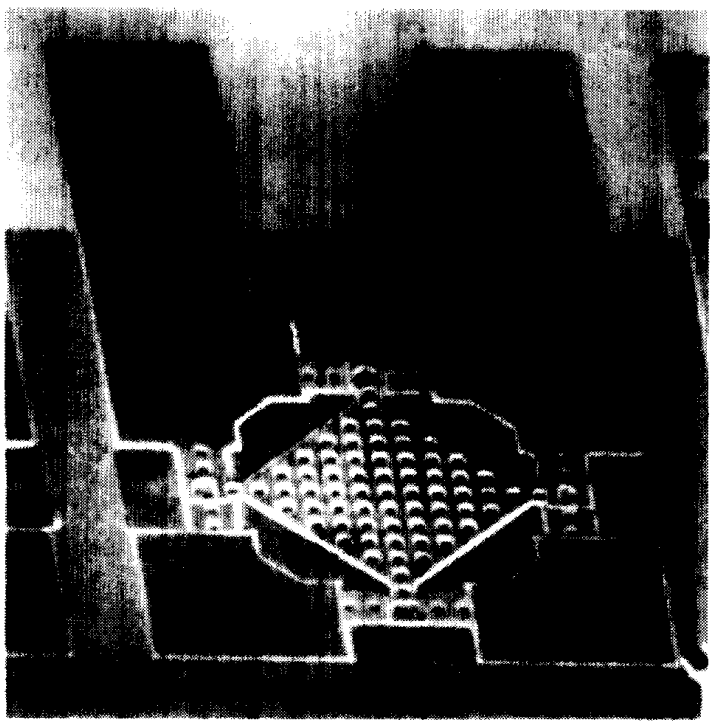

Fig. 3. A close-up SEM view of a force sensor.

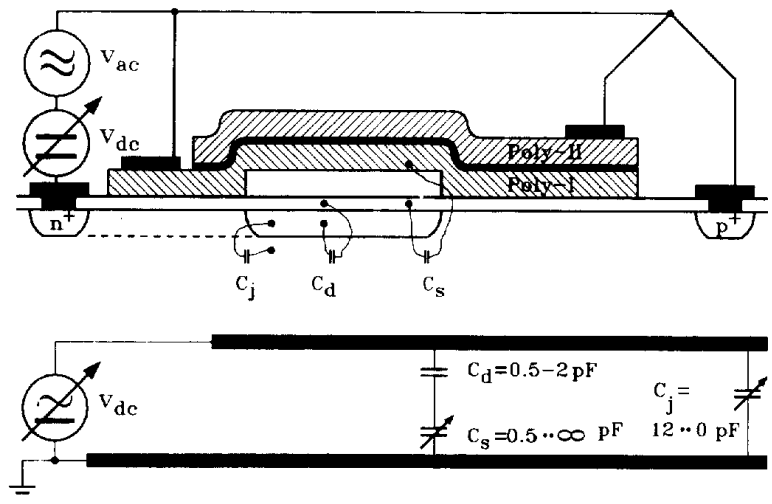

Fig. 4. The electrostatic pull-in voltage experiment and an overview of the most important capacitances in the experiment.

structure. The mechanical force $F_{\text {mech }}$ and the electrostatic force $F_{\text {electr }}$ can be extracted from the mechanical energy of the spring element $U_{\text {mech }}$ and from the electrostatic energy stored in the sensor/dielectric capacitor structure $U_{\text {electr }}$ :

$$
\begin{gathered}
U_{\text {med } \mathrm{t}}=\frac{1}{2} k \delta^{2} \\
U_{\text {electr }}=\frac{1}{2}\left[\frac{\varepsilon_{o} A}{d_{o}-\delta+\left(d_{d} / \varepsilon_{r}\right)}\right] V^{2}
\end{gathered}
$$

with

$k=$ spring constant of sensor: $k=1.6 \times 10^{3} \mathrm{~N} / \mathrm{m}$.

$\delta=$ displacement of membrane from initial state.

$\varepsilon_{0}=$ electric permittivity: $\varepsilon_{0}=8.8 \times 10^{-12} \mathrm{~s}^{2} \mathrm{C}^{2} / \mathrm{m}^{3} \cdot \mathrm{kg}$.

$\varepsilon_{r}=$ relative permittivity of dielectric medium.

$A=$ membrane area: $A=150 \times 150 \cdots 175 \times 175 \mu \mathrm{m}^{2}$.

$V=$ applied dc voltage: $V=0-30 \mathrm{~V}$.

$d_{o}=$ initial capacitive gap separation: $d_{0}=0.5 \mu \mathrm{m}$.

$d_{d}=$ thickness of dielectric layer: $d_{d}=0.2-0.5 \mu \mathrm{m}$.

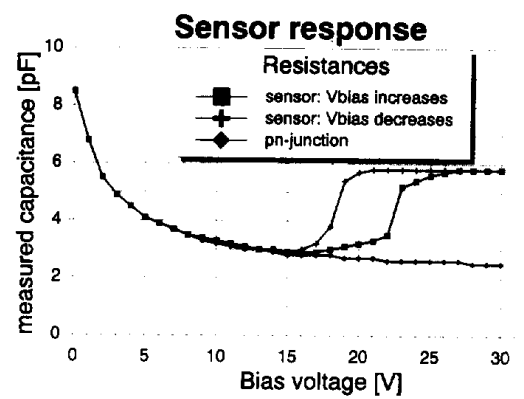

Fig. 5. The measured change in capacitance versus applied voltage.

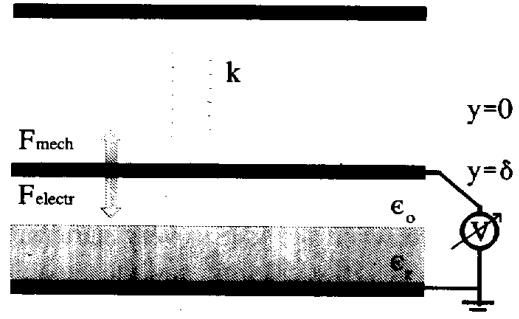

Fig. 6. Overview of forces on a membrane.

$C_{0}=$ initial sensor capacitance.

$C_{d}=$ capacitance of dielectric layer.

The forces acting on the membrane are then as follows:

$$
\begin{gathered}
F_{\text {mech }}=\left[\frac{d U_{\text {mech }}}{d y}\right]_{y=\delta}=k \delta \\
F_{\text {electr }}=\left[\frac{d U_{\text {electr }}}{d y}\right]_{y=\delta}=\frac{-\frac{1}{2} \varepsilon_{o} A V^{2}}{\left[d_{o}-\delta+\left(d_{d} / \varepsilon_{r}\right)\right]^{2}}
\end{gathered}
$$

and, with $F_{\text {mech }}+F_{\text {electr }}=0$, the displacement to voltage is given by

$$
\begin{aligned}
V & =2 \sqrt{\frac{F_{\max } \cdot \delta}{C_{o}}}\left(1-\frac{\delta}{d_{o}}+\frac{C_{o}}{C_{d}}\right) \text { with } F_{\max } \\
& =k \frac{d_{o}}{2} .
\end{aligned}
$$

Fig. 7 is a graphical representation of (6). The pull-in voltage $V_{\mathrm{pi}}$ is at $\partial V /\left.\partial \delta\right|_{F}=0$, and the pull-out voltage $V_{\mathrm{po}}$ is at $V\left(\delta=d_{o}\right)$. Using $V_{\mathrm{pi}}=23 \mathrm{~V}$ and $V_{\mathrm{po}}=18 \mathrm{~V}$, the force range and dielectric capacitance are found from Fig. 7, which are $F_{\max }=0.21 \mathrm{mN}$ and $C_{d}=0.8 \mathrm{pF}$. This is in agreement with the prediction in (1).

A small size of a force-sensing element for tactile sensor applications is not a serious problem. The sensor can be integrated with a bipolar process on the same chip. The charge amplifier functions as a mechanical-to-electric interface module with the sensor signal and has been designed with a minimum number of transistors. Experiments with the system for analog readout demonstrated the capability of the system to detect changes in a capacitance of less than $5 \mathrm{fF}$, and the nonlinearity in the response is $0.25 \%$ in the $0.5-1.0 \mathrm{pF}$ region. This results in sensitivity for the sensor of $1-5 \mathrm{fF} / \mu \mathrm{N}$. 


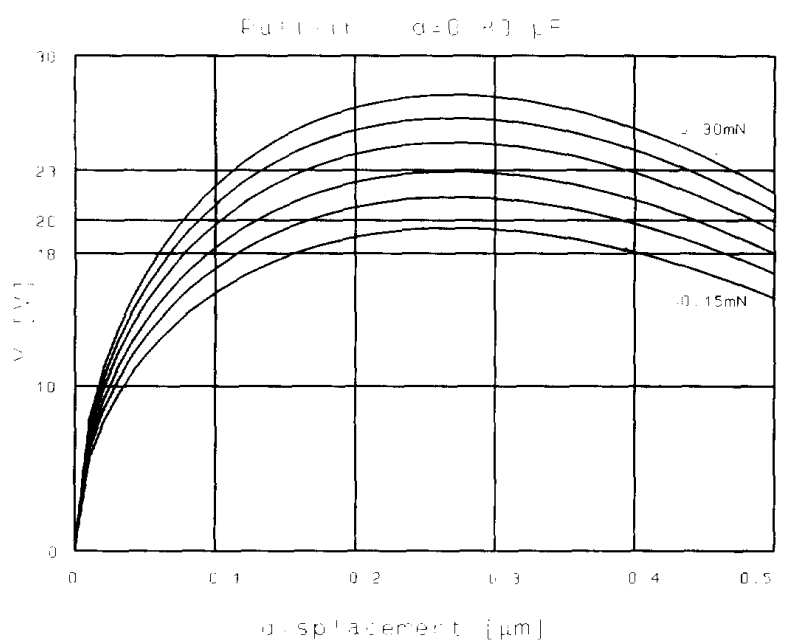

Fig. 7. The simulated pull-in voltage experiment: $C_{d}=0.8 \mathrm{pF}$ and $F_{\max }=$ $0.15-0.3 \mathrm{mN}$.

\section{CONCLUSIONS}

A surface micromachined capacitive sensor for the detection of microforces has been presented. The sensor operation has been evaluated, and basic parameters like force range and sensitivity have been calculated. Experiments have been presented, which show that the calculated performance is in agreement with practical force sensing structures. The sensor has been applied in a tactile matrix for the acquisition of object features when touching this object.

\section{ACKNOWLEDGMENT}

The authors are indebted to members of DIMES for their skillful fabrication of the bipolar circuits. They would like to thank Y. X. Li and P. J. French of the Electronic Instrumentation Laboratory for the sensor fabrication.

\section{REFERENCES}

[1] Y. X. Li et al., "Reactive ion etching (RIE) techniques for micromachining applications," presented at Eurosensors VII, Budapest, Sept. 26-29, 1993. Published in Sensors and Actuators, vol. A41-42, pp. 317-321, 1994.

[2] K. Suzuki, K. Nafjafi, and K. D. Wise, "A 1024-element highperformance silicon tactile imager," IEEE Trans. Electron Devices, vol. 37 , no. 8 , pp. $1852-1860,1990$.

[3] M. R. Wolffenbuttel et al., "Multilayer membranes for a tactile imaging sensor using surface micromachining and RIE," in Proc. 7th Int. Conf. Solid-State Sensors and Actuators (TRANSDUCERS '93), Yokohama, Japan, June 7-10, 1993, pp. 284-287.

[4] S. Timoshenko, Strength of Materials, Part II: Advanced Theory and Problems, New York: van Nostrand, 3rd ed, 1978.

[5] M. R. Wolffenbuttel, P. P. L. Regtien, and R. F. Wolffenbuttel, "The read-out characteristics of an integrated charge amplifier for potentially broad-field applications," in Proc. 1992 IEEE Int. Symp. Circuits and Systems (ISCAS '92, San Diego, CA, May 10-13, 1992, pp. 2356-2359. [6] D. W. de Bruin, H. V. Allen, and S. C. Terry, "Second-order effects in self-testable accelerometers," in Proc. IEEE Solid-State Sensor and Actuator Workshop 1990, Hilton Head Island, SC, June 4-7, 1990, pp. 149-152. 